\title{
Discordance Between Apolipoprotein B and Low-Density Lipoprotein Cholesterol and Progression of Coronary Artery Calcification in Middle Age
}

\author{
Chan-Won Kim, MD, PhD; Sungwoo Hong, MD; Yoosoo Chang, MD, PhD; \\ Jung Ah Lee, MD, PhD; Hocheol Shin, MD, PhD; Seungho Ryu, MD, PhD
}

\begin{abstract}
Background: A high level of apolipoprotein $B(a p o B)$ is associated with incident coronary artery disease (CAD) when low-density lipoprotein cholesterol (LDL-C) level is discordantly low or concordantly high. However, data on the relationship of apoB with subclinical measure of $C A D$ are limited.

Methods and Results: A total of 14,205 men (mean age 41.0 years) who were free of cardiovascular disease at baseline and who underwent a health checkup exam, including measurement of coronary artery calcium (CAC), were studied. Of the study group, 2,773 participants (19.5\%) had CAC at baseline, and CAC progression was observed in 2,550 (18.0\%). The multivariate-adjusted CAC score ratios (95\% confidence interval) comparing discordantly high apoB/low LDL-C and concordantly high apoB/high LDL-C with concordantly low apoB/low LDL-C were $1.51(0.98-2.32)$ and $2.70(2.19-3.33)$, respectively. The corresponding relative risks for CAC progression were $1.26(1.02-1.56)$ and $1.49(1.34-1.66)$, respectively. These associations did not change appreciably after adjustment for insulin resistance and subclinical inflammation.
\end{abstract}

Conclusions: Discordant analysis showed that a high apoB level was strongly associated with prevalence and progression of CAC independent of LDL-C in a large cohort of healthy adults. The present study results highlighted the importance of an apoB measure as a potential target for primary prevention of coronary atherosclerosis in healthy adults.

Key Words: Apolipoproteins; Atherosclerosis; Cholesterol; Coronary artery disease

A rterial retention of apolipoprotein B (apoB)-containing particles plays a critical role in the pathogenesis of atherosclerosis. ${ }^{1}$ To evaluate the atherogenic risk attributable to apoB particles, measures of low-density lipoprotein cholesterol (LDL-C), non-high-density lipoprotein cholesterol (HDL-C), and apoB have been proposed. ${ }^{2}$ The first two measures account for the mass of cholesterol within LDL, intermediate density lipoprotein (IDL), lipoprotein (a) (Lp (a)), or very low-density lipoprotein (VLDL) particles. A measure of apoB is the sum of the number of LDL, IDL, Lp(a), and VLDL particles, because each of these lipoprotein particles contains one molecule of apoB. ${ }^{2}$ Although the levels of apoB in most people are highly correlated with those of LDL-C (concordant), cholesterol mass associated with apoB is either greater or lesser than the average cholesterol mass (discordant) in $10-30 \%$ of individuals. ${ }^{3,4}$ These features can explain why conventional analyses, which treated these 2 measures as independent variables, have produced largely negative results in evaluating the predictive superiority of one measure over another. ${ }^{5}$ Accordingly, it is of paramount importance to evaluate differential effects of apoB and LDL-C on the study outcome using discordant analysis, which analyzes these highly correlated factors by categorizing their distribution into concordant or discordant groups. A high correlation between measures in concordant groups is less likely to affect the predictive ability than in the discordant groups. ${ }^{4}$

Multiple epidemiological studies using discordant analysis showed that apoB predicts residual risk in patients with optimal control of LDL-C. ${ }^{67}$ Moreover, a recent Mendelian

Received June 25, 2020; revised manuscript received September 22, 2020; accepted October 8, 2020; J-STAGE Advance Publication released online December 11, 2020 Time for primary review: 28 days

Center for Cohort Studies, Total Healthcare Center (C.-W.K., S.H., Y.C., J.A.L., S.R.), Workplace Health Institute, Total Healthcare Center (C.-W.K., S.H., J.A.L.), Department of Occupational Medicine (Y.C., S.R.), Department of Family Medicine (H.S.), Kangbuk Samsung Hospital, Sungkyunkwan University, School of Medicine, Seoul; Department of Clinical Research Design \& Evaluation, SAIHST, Sungkyunkwan University, Seoul (Y.C., S.R.), Korea

The first two authors are joint first authors (C.-W.K., S.H.).

Mailing address: Chan-Won Kim, MD, PhD, Center for Cohort Studies, Total Healthcare Center, Kangbuk Samsung Hospital, Sungkyunkwan University School of Medicine, Samsung Main Building B2, 67 Sejong-daero, Jung-gu, Seoul 04514, Republic of Korea. E-mail: chanwon.kim75@gmail.com

All rights are reserved to the Japanese Circulation Society. For permissions, please e-mail: cj@j-circ.or.jp

ISSN-1346-9843 
randomization study showed that risk of cardiovascular events was determined by a concentration of apoB-containing lipoprotein particles rather than by total cholesterol (TC) mass. ${ }^{8}$ Hence, a measure of apoB is of considerable significance for predicting coronary artery disease (CAD) events beyond a measure of LDL-C. ${ }^{9}$ Nevertheless, few studies have quantified apoB as an early marker of coronary atherosclerosis in a population-based study. Coronary artery calcium (CAC) score is a subclinical measure of calcified atherosclerotic plaque burden used to predict the development of coronary events. ${ }^{10}$ Progression of CAC can be helpful as an intermediate outcome in the prediction of cardiovascular disease events in healthy middle-aged adults not likely to experience a clinical CAD event. ${ }^{11}$

Therefore, we examined the association between LDL-C and apoB with the presence and progression of $\mathrm{CAC}$ in a large sample of young and middle-aged asymptomatic men and woman participating in a health screening program.

\section{Methods}

\section{Subjects}

The Kangbuk Samsung Health Study is an ongoing cohort study of adults who underwent comprehensive annual or biennial examinations at the clinics of the Kangbuk Samsung Hospital Screening Center in Seoul and Suwon, South Korea, from 2002 to the present. ${ }^{12}$ More than $80 \%$ of the examinees were employees of various companies or local governmental organizations. In South Korea, annual or biennial health screening exams are widely performed, as they are mandatory for all workers under the Industrial Safety and Health Law. The remaining examinees voluntarily paid for screening exams. The study population consists of 14,574 Korean men who underwent a comprehensive health check-up examination including CAC measurement on 2 occasions between March 2011 and December 2016. For our data analyses, we excluded participants with CAD $(n=53)$, those with cerebrovascular disease $(n=33)$, and those with missing data on baseline lipid measures $(n=289)$. The total number of eligible participants for this study was 14,205 . This study was approved by the Institutional Review Board of Kangbuk Samsung Hospital (reference number: 2018-06-016), and informed consent was waived because researchers only retrospectively accessed a deidentified database for analysis purposes.

\section{Exposure Assessment}

Data on medical history, medication use, family history, smoking history, physical activity, alcohol consumption, and education level were collected through a self-administered questionnaire. Smoking status was categorized into 3 levels: never, former, and current smoker. Excessive alcohol consumption was defined as an average daily alcohol intake $\geq 20 \mathrm{~g}$. Physical activity level was assessed using the Korean-validated short version of the International Physical Activity Questionnaire (IPAQ) and was defined as weekly energy expenditure in metabolic equivalent (MET) hours per week. Body weight was measured to the nearest $0.1 \mathrm{~kg}$ using a digital scale with the patient wearing light clothing and no shoes, and height was measured by trained nurses. Body mass index (BMI) was calculated as weight/ height squared $\left(\mathrm{kg} / \mathrm{m}^{2}\right)$, and obesity was defined as a BMI of 25 or higher according to Asian-specific criteria. Depression was assessed by using the Korean version of the Center for Epidemiologic Studies Depression (CES-D) Scale.
Blood pressure (BP) was measured using an automated oscillometric device (Model 53000; Welch Allyn, New York, USA) while participants were in a seated position with the arm supported at heart level. Three BP readings were recorded for each participant, and the average of the second and third readings was used in the analyses to reduce measurement error. Hypertension was defined as systolic $\mathrm{BP} \geq 140 \mathrm{mmHg}$, diastolic $\mathrm{BP} \geq 90 \mathrm{mmHg}$, or current use of antihypertensive medication. Blood samples were collected after an at least 10 -h fast. The methods for measuring serum levels of high sensitive $\mathrm{C}$-reactive protein (hsCRP), TC, LDL-C, triglycerides (TG), and HDL-C have been reported elsewhere. ${ }^{13} \mathrm{ApoB}$ was measured using Roche diagnostics reagent kits on an automated chemistry analyzer (Modular DPP; Roche Diagnostics, Tokyo, Japan). Serum insulin was measured using an electrochemiluminescence immunoassay on a Modular Analytics E170 apparatus (Roche Diagnostics, Tokyo, Japan). Insulin resistance was assessed with the homeostasis model assessment of insulin resistance (HOMA-IR): fasting serum glucose (FSG; $\mathrm{mg} / \mathrm{dL}) \times$ fasting insulin $(\mu \mathrm{IU} / \mathrm{mL}) / 405$. We used the low serum LDL-C/apoB ratio as an indirect measure of LDL particle size, and fasting remnant cholesterol was calculated as TC minus LDL-C minus HDL-C.

\section{Outcomes}

The CAC was measured using a Lightspeed VCT XTe-64 slice multidetector computed tomography (CT) scanner (GE Healthcare, Tokyo, Japan) following the same protocol in both study centers. CT scans were obtained using a standard procedure with slice thickness, $2.5 \mathrm{~mm}$; rotation time, $400 \mathrm{~ms}$; tube voltage, $120 \mathrm{kV}$; and tube current, $124 \mathrm{mAs}(310 \mathrm{~mA} \times 0.4 \mathrm{~s})$ under electrocardiogram-gated dose modulation. CAC scores were calculated by using the method described in Agatston et al. ${ }^{14}$ The presence of CAC was defined as a CAC score $>0$, and CAC progression was defined as follows: CAC score $>0$ at follow-up examination among those who had no CAC at baseline; change in CAC score/year $\geq 10$ at follow up for those with $0<$ baseline $\mathrm{CAC}<100$; or a percentage change in CAC score (annualized change in CAC score divided by that at baseline) $\geq 10 \%$ at follow up for those who had baseline a CAC score $\geq 100 .{ }^{15}$ Intra- and inter-observer reliability of the CAC scores was excellent (intra-class correlation coefficient, 0.99 ).

\section{Statistical Analyses}

The characteristics of participants are displayed as mean and standard deviation for continuous variables with normal distribution, interquartile ranges for continuous variables with skewed distribution, and percentages for categorical variables. Participants were classified into low, middle, and high tertile groups based on apoB and LDL-C measures. After computing the median values of apoB and LDL-C, we categorized the participants into the following 4 groups: (1) both apoB and LDL-C measures less than the corresponding median values; (2) apoB measure less than its median value and LDL-C measure greater than or equal to its median value; (3) apoB measure greater than or equal to its median value and LDL-C measure less than its median value; and (4) both apoB and LDL-C measures greater than the corresponding median values (Figure). The association between discordance/concordance of apoB and LDL-C and CAC progression was evaluated using Poisson regression with robust error variance estimation to compute risk ratio (RR) and the $95 \%$ confidence interval 


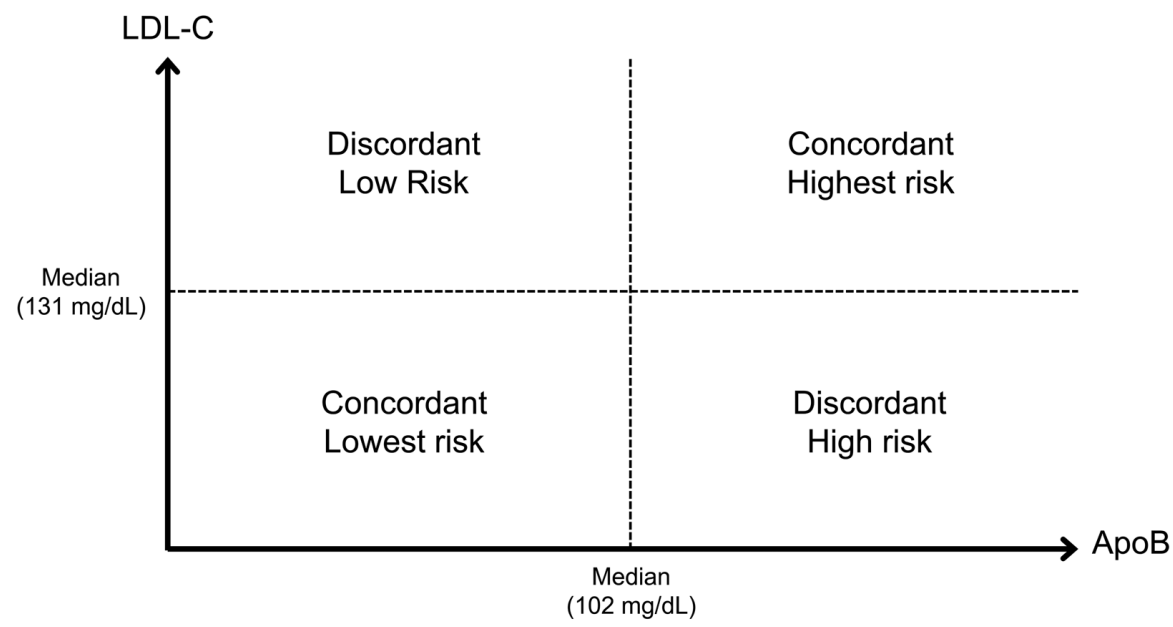

Figure. Quadrant plot of low-density lipoprotein-cholesterol (LDL-C) vs. apolipoprotein B (ApoB). Median values were LDL-C $131 \mathrm{mg} / \mathrm{dL}$ and ApoB $102 \mathrm{mg} / \mathrm{dL}$.

\begin{tabular}{|c|c|c|c|c|}
\hline \multirow{2}{*}{ Characteristics } & \multicolumn{3}{|c|}{ ApoB tertile (range) } & \multirow{2}{*}{ P value* } \\
\hline & Low (22-92) & Middle (93-113) & High (114-251) & \\
\hline No. & 4,756 & 4,846 & 4,603 & \\
\hline Age, years & $40.5(6.2)$ & $41.2(6.1)$ & $41.4(5.8)$ & $<0.001$ \\
\hline Seoul center, \% & 43.2 & 47.0 & 50.4 & $<0.001$ \\
\hline Education, \%A & 88.5 & 87.8 & 86.9 & 0.089 \\
\hline Depression, \% ${ }^{\mathrm{B}}$ & 6.9 & 6.7 & 7.3 & 0.636 \\
\hline Current smoking, \% & 31.1 & 32.5 & 37.1 & $<0.001$ \\
\hline Alcohol intake, \%c & 16.4 & 15.1 & 12.8 & $<0.001$ \\
\hline HEPA, \% & 16.4 & 15.1 & 12.8 & $<0.001$ \\
\hline $\mathrm{BMI}, \mathrm{kg} / \mathrm{m}^{2}$ & $24.1(2.9)$ & $25.0(2.9)$ & $25.7(2.9)$ & 0.096 \\
\hline $\mathrm{WC}, \mathrm{cm}$ & $85.0(8.0)$ & $87.5(7.6)$ & $89.2(7.3)$ & $<0.001$ \\
\hline Systolic BP, mmHg & $114.0(11.6)$ & $115.4(12.0)$ & $116.6(12.3)$ & $<0.001$ \\
\hline Diastolic BP, mmHg & $74.1(9.3)$ & $75.7(9.6)$ & $77.1(9.9)$ & $<0.001$ \\
\hline Hypertension, \% & 17.5 & 19.6 & 21.2 & $<0.001$ \\
\hline $\mathrm{FSG}, \mathrm{mg} / \mathrm{dL}$ & $98.2(14.6)$ & $99.1(15.4)$ & $101.7(21.6)$ & $<0.001$ \\
\hline HOMA-IR & $1.2(0.8-1.8)$ & $1.4(1.0-2.1)$ & $1.7(1.2-2.6)$ & $<0.001$ \\
\hline Diabetes, \% & 7.2 & 5.5 & 7.9 & $<0.001$ \\
\hline $\mathrm{TC}, \mathrm{mg} / \mathrm{dL}$ & $173.8(22.4)$ & $203.7(19.2)$ & $239.6(27.9)$ & $<0.001$ \\
\hline Triglycerides, mg/dL & $97.0(73.0-137.0)$ & $129.0(96.0-176.0)$ & $164.0(121.0-223.0)$ & $<0.001$ \\
\hline LDL-C, mg/dL & $100.5(18.0)$ & $130.3(14.9)$ & $163.6(23.9)$ & $<0.001$ \\
\hline HDL-C, mg/dL & $55.0(13.8)$ & $50.6(11.8)$ & $48.3(10.3)$ & $<0.001$ \\
\hline hsCRP, mg/dL & $0.05(0.03-0.09)$ & $0.06(0.03-0.11)$ & $0.07(0.04-0.13)$ & $<0.001$ \\
\hline \multicolumn{5}{|l|}{ CAC } \\
\hline 0 & 86.0 & 81.6 & 73.5 & $<0.001$ \\
\hline$>0$, Agatston unit & $21.0(7.0-65.0)$ & $17.0(4.0-48.0)$ & $18.0(5.0-53.0)$ & $<0.001$ \\
\hline
\end{tabular}

Data are presented as mean (standard deviation), median (interquartile range), or percentage. ${ }^{A} A t$ least a college graduate. ${ }^{B}$ The Center for Epidemiologic Studies Depression (CES-D) score of 16 or higher. ${ }^{C}$ Alcohol intake of $\geq 20 \mathrm{~g} / \mathrm{day}$. ApoB, Apolipoprotein B; BMI, body mass index; BP, blood pressure; CAC, coronary artery calcium; FSG, fasting serum glucose; HDL-C, high density lipoprotein cholesterol; HEPA, health enhancing physical activity; HOMA-IR, homeostasis model assessment of insulin resistance; hsCRP, high sensitivity C-reactive protein; LDL-C, low-density lipoprotein cholesterol; TC, total cholesterol; WC, waist circumference. ${ }^{*} P$ values derived from $X^{2}$ test for categorical variables and 1-way analysis of variance test for continuous variables. 


\begin{tabular}{|c|c|c|c|c|c|}
\hline & \multicolumn{4}{|c|}{ Concordance/discordance groups (apoB and LDL-C) } & \multirow[b]{2}{*}{ P value } \\
\hline & $\begin{array}{l}\text { Low apoB/ } \\
\text { Low LDL-C }\end{array}$ & $\begin{array}{l}\text { Low apoB/ } \\
\text { High LDL-C }\end{array}$ & $\begin{array}{l}\text { High apoB/ } \\
\text { Low LDL-C }\end{array}$ & $\begin{array}{l}\text { High apoB/ } \\
\text { High LDL-C }\end{array}$ & \\
\hline No. & 6,257 & 1,113 & 751 & 6,084 & \\
\hline Age, years & $40.8(6.2)$ & $40.4(6.2)$ & $41.7(6.0)$ & $41.3(5.9)$ & $<0.001$ \\
\hline Seoul center, \% & 44.4 & 41.8 & 54.3 & 49.3 & $<0.001$ \\
\hline Education, \%A & 88.1 & 89.9 & 84.8 & 87.4 & 0.010 \\
\hline Depression, \% ${ }^{\mathrm{B}}$ & 6.8 & 5.7 & 9.9 & 7.0 & 0.009 \\
\hline Current smoking, \% & 32.0 & 27.1 & 40.6 & 35.3 & $<0.001$ \\
\hline Alcohol intake, $\%$ c & 32.2 & 28.7 & 45.2 & 34.9 & $<0.001$ \\
\hline HEPA, \% & 15.9 & 17.4 & 14.3 & 13.2 & $<0.001$ \\
\hline $\mathrm{BMI}, \mathrm{kg} / \mathrm{m}^{2}$ & $24.3(3.0)$ & $24.5(2.9)$ & $25.8(2.8)$ & $25.5(2.9)$ & 0.016 \\
\hline Obesity, \% & 34.0 & 38.7 & 57.8 & 52.0 & $<0.001$ \\
\hline $\mathrm{WC}, \mathrm{cm}$ & $85.7(8.0)$ & $86.0(7.6)$ & $89.7(7.1)$ & $88.7(7.4)$ & $<0.001$ \\
\hline Systolic BP, mmHg & $114.1(11.7)$ & $115.9(11.7)$ & $117.6(12.9)$ & $116.2(12.2)$ & $<0.001$ \\
\hline Diastolic BP, mmHg & $74.5(9.4)$ & $74.6(9.2)$ & $78.5(10.5)$ & $76.5(9.8)$ & $<0.001$ \\
\hline Hypertension, \% & 18.6 & 14.1 & 31.4 & 19.7 & $<0.001$ \\
\hline $\mathrm{FSG}, \mathrm{mg} / \mathrm{dL}$ & $98.6(15.2)$ & $97.3(12.8)$ & $105.2(26.0)$ & $100.4(18.9)$ & $<0.001$ \\
\hline HOMA-IR & $1.3(0.9-1.9)$ & $1.1(0.8-1.7)$ & $2.0(1.4-2.8)$ & $1.6(1.1-2.4)$ & $<0.001$ \\
\hline Diabetes, \% & 6.8 & 3.3 & 12.1 & 6.4 & $<0.001$ \\
\hline $\mathrm{TC}, \mathrm{mg} / \mathrm{dL}$ & $177.1(21.1)$ & $211.7(16.8)$ & $201.7(28.9)$ & $233.6(26.6)$ & $<0.001$ \\
\hline Triglycerides, mg/dL & $106.0(77.0-153.0)$ & $106.0(83.0-140.0)$ & $210.0(141.0-304.0)$ & $150.0(112.0-203.0)$ & $<0.001$ \\
\hline LDL-C, mg/dL & $105.3(17.1)$ & $139.5(8.5)$ & $121.5(9.2)$ & $160.6(22.3)$ & $<0.001$ \\
\hline HDL-C, mg/dL & $52.0(43.0-61.0)$ & $54.0(47.0-62.0)$ & $42.0(36.0-49.0)$ & $48.0(42.0-55.0)$ & $<0.001$ \\
\hline Non HDL-C, mg/dL & $123.8(21.1)$ & $156.3(15.0)$ & $158.3(29.1)$ & 184. (26.0) & $<0.001$ \\
\hline ApoB, mg/dL & $83.3(12.6)$ & $97.3(5.2)$ & $110.0(6.6)$ & $124.9(6.6)$ & $<0.001$ \\
\hline LDL-C/apoB ratio & $1.27(0.14)$ & $1.44(0.12)$ & $1.11(0.10)$ & $1.29(0.10)$ & $<0.001$ \\
\hline Remnant-C, mg/dL & $16.0(10.0-23.0)$ & $16.0(11.0-22.0)$ & $29.0(20.0-43.0)$ & $22.0(15.0-30.0)$ & $<0.001$ \\
\hline Medication for dyslipidemia, \% & 5.2 & 0.9 & 8.4 & 3.4 & $<0.001$ \\
\hline \multicolumn{6}{|l|}{ CAC } \\
\hline 0 & 84.3 & 86.3 & 75.4 & 76.1 & $<0.001$ \\
\hline$>0$, Agatston unit & $21.0(6.0-62.0)$ & $12.5(3.0-42.0)$ & $15.0(6.0-48.0)$ & $17.0(5.0-52.0)$ & $<0.001$ \\
\hline
\end{tabular}

Data are presented as mean (standard deviation), median (interquartile range), or percentage. ${ }^{A} A t$ least a college graduate. ${ }^{B}$ The Center for Epidemiologic Studies Depression (CES-D) score of 16 or higher. ${ }^{C}$ Alcohol intake of $\geq 20 \mathrm{~g} /$ day. Remnant-C, Remnant cholesterol. Other abbreviations as in Table 1. ${ }^{*} P$ values derived from $X^{2}$ test for categorical variables and 1-way analysis of variance test for continuous variables.

(CI). We used 3 multivariate models with progressive adjustments: Model 1 was adjusted for age, sex, year of examination, and study center (Seoul or Suwon); Model 2 was further adjusted for family history of CAD, education, marital status, employment status (yes/no), depressive symptom scores, antidepressant use, smoking status, alcohol consumption, and physical activity level (inactive, minimally active, health-enhancing physical activity [HEPA], or unknown); and Model 3 was further adjusted for systolic BP, diastolic BP, antihypertensive medication, history of type 2 diabetes mellitus, HDL-C, and lipid-lowering medication use. To evaluate the dose-response relationship, we also modeled the association between lipid measure and risk of CAC progression using a restricted cubic spline model. All $\mathrm{P}$ values were 2-tailed, and $\mathrm{P}$ values $<0.05$ were considered statistically significant. We used STATA version 14.0 (Stata Corp., College Station, TX, USA) for all data analyses.

\section{Results}

The demographic, lifestyle behaviors, and cardio-metabolic features of participants at baseline are summarized in
Table 1. The average age of the study participants was 41.0 years. Participants in the low tertile of apoB were more likely to have a HEPA level and less likely to be current smokers and to have hypertension compared with those in the middle or high apoB tertiles. The high tertile of apoB had participants with the highest baseline BMI, BP, FSG, TC, LDL-C, TG, hsCRP, and HOMA-IR levels. Education and depressive symptoms were not significantly related to the apoB tertile.

The baseline characteristics of the 4 concordance/discordance groups, into which the participants were categorized according to apoB and LDL-C levels (concordantly low apoB/low LDL-C, discordantly low apoB/high LDL-C, discordantly high apoB/low LDL-C, and concordantly high apoB/high LDL-C), are presented in Table 2. The proportion of participants with discordantly low and discordantly high apoB was $7.8 \%$ and $5.3 \%$, respectively. Participants in the higher apoB groups tended to have unhealthy lifestyle behaviors, such as a current smoking status, excessive alcohol consumption, and lower physical activity. Likewise, BMI, waist circumference, BP, and glucose parameters were higher in the high apoB groups than in the low apoB groups. As expected, unfavorable risk 


\begin{tabular}{|c|c|c|c|c|}
\hline \multirow[b]{3}{*}{ ApoB } & \multicolumn{4}{|c|}{ Multivariate CAC score ratios ( $95 \%$ confidence interval) } \\
\hline & Crude & Model 1 & Model 2 & Model 3 \\
\hline & & & & \\
\hline First tertile & 1.00 & 1.00 & 1.00 & 1.00 \\
\hline Second tertile & $2.13(1.61-2.81)$ & $1.66(1.29-2.14)$ & $1.88(1.47-2.40)$ & $1.63(1.27-2.09)$ \\
\hline Third tertile & $7.01(5.38-9.12)$ & $4.50(3.53-5.74)$ & $4.98(3.91-6.33)$ & $3.93(3.06-5.04)$ \\
\hline \multicolumn{5}{|l|}{ LDL-C } \\
\hline First tertile & 1.00 & 1.00 & 1.00 & 1.00 \\
\hline Second tertile & $1.46(1.11-1.92)$ & $1.45(1.13-1.85)$ & $1.81(1.42-2.30)$ & $1.63(1.28-2.07)$ \\
\hline Third tertile & $3.14(2.42-4.09)$ & $2.89(2.28-3.66)$ & $3.76(2.97-4.76)$ & $3.24(2.56-4.10)$ \\
\hline \multicolumn{5}{|l|}{ Non-HDL-C } \\
\hline First tertile & 1.00 & 1.00 & 1.00 & 1.00 \\
\hline Second tertile & $1.60(1.22-2.11)$ & $1.63(1.28-2.08)$ & $1.86(1.46-2.37)$ & $1.62(1.27-2.07)$ \\
\hline Third tertile & $3.31(2.54-4.31)$ & $3.46(2.73-4.39)$ & $3.92(3.10-4.96)$ & $3.07(2.40-3.92)$ \\
\hline \multicolumn{5}{|l|}{ ApoB/LDL-C } \\
\hline Low apoB/Low LDL-C & 1.00 & 1.00 & 1.00 & 1.00 \\
\hline Low apoB/High LDL-C & $0.59(0.37-0.93)$ & $0.98(0.64-1.51)$ & $1.33(0.88-2.03)$ & $1.36(0.90-2.07)$ \\
\hline High apoB/Low LDL-C & $3.94(2.48-6.26)$ & $2.49(1.63-3.82)$ & $2.05(1.34-3.13)$ & $1.51(0.98-2.32)$ \\
\hline High apoB/High LDL-C & $3.61(2.87-4.55)$ & $2.84(2.30-3.49)$ & $3.22(2.62-3.96)$ & $2.70(2.19-3.33)$ \\
\hline \multicolumn{5}{|l|}{ ApoB/Non-HDL-C } \\
\hline Low apoB/Low non-HDL-C & 1.00 & 1.00 & 1.00 & 1.00 \\
\hline Low apoB/High non-HDL-C & $0.66(0.43-1.00)$ & $1.03(0.69-1.53)$ & $1.22(0.83-1.79)$ & $1.06(0.72-1.56)$ \\
\hline High apoB/Low non-HDL-C & $6.21(3.58-10.75)$ & $2.61(1.58-4.32)$ & $2.46(1.49-4.06)$ & $2.15(1.31-3.55)$ \\
\hline High apoB /High non-HDL-C & $3.50(2.78-4.41)$ & $2.83(2.30-3.49)$ & $3.10(2.52-3.81)$ & $2.50(2.02-3.10)$ \\
\hline
\end{tabular}

†CAC ratios derived from Tobit regression models using $\ln (C A C+1)$ as the outcome. Multivariable model 1 was adjusted for age, year of screening examination, and study center; model 2 was further adjusted for family history of coronary artery disease, education, diabetes, hypertension, smoking status, alcohol consumption, physical activity, and medication for dyslipidemia; model 3 was further adjusted for systolic BP, diastolic BP, BMI, and HDL-C. Abbreviations as in Table 1.

factor profiles were higher in participants with concordantly high apoB/high LDL-C than in those with concordantly low apoB/low LDL-C. However, interestingly and unexpectedly, we found that the discordantly low apoB/high LDL-C group had the lowest prevalence of hypertension and diabetes, and the discordantly high apoB/low LDL-C group had the highest prevalence of both hypertension and diabetes and the lowest levels of HDL-C. The discordantly high apoB/low LDL-C group had the lowest LDL-C/apoB ratio and the highest fasting remnant cholesterol.

In Table 3, individuals in the middle and the high tertiles of apoB had a higher prevalence of coronary calcification than those in the lowest tertile (adjusted CAC score ratios for coronary calcification: $1.63,95 \%$ CI $1.27-2.09$ in the middle tertile; 3.93 , 95\% CI 3.06-5.04 in the high tertile). We observed similar results across tertiles of LDL-C. The results of a cross-sectional analysis showed that the prevalence of a positive CAC score was significantly higher than that of the reference (low apoB/low LDL-C) when LDL-C was either high or low and apoB was high.

Median follow-up duration was 2.6 years, and CAC progression was found in 2,550 participants $(18.0 \%)$. Within the study group, 2,773 participants $(19.5 \%)$ had CAC at baseline, whereas 835 of these $(7.3 \%)$ developed incident $\mathrm{CAC}$ during the study period. The associations of apoB and LDL-C tertiles and CAC progression are shown in Table 4. Compared with the lowest tertile (reference), the relative risk of CAC progression for the middle and high apoB tertiles was 1.14 (95\% CI, 1.00-1.29) and 1.64 (95\% CI, 1.44-1.85), respectively, and adjustment for relevant behaviors, comorbidities, and biological factors did not appreciably change these associations. By using these covariates at baseline, the adjusted relative risk for CAC progression comparing high apoB/low LDL-C and high apoB/high LDL-C with the reference (low apoB/low LDL-C) was 1.26 (95\% CI, 1.02$1.56)$ and 1.49 (95\% CI, 1.34-1.66), respectively. These associations were similar across concordance and discordance between apoB and non HDL-C.

\section{Discussion}

In this large study of young and middle-aged adults, apoB and LDL-C demonstrated a dose-response relationship with both incidence and progression of CAC, independent of demographic variables, relevant behaviors, and cardiovascular risk factors. In the fully adjusted model, the discordant high-risk group (high apoB/high LDL-C) and the concordant highest-risk group were associated with higher risk for CAC incidence and progression than the reference group (low apoB/low LDL-C), whereas there was no significant difference in the risk between the discordant low-risk group (low apoB/high LDL-C) and the reference group. These findings suggest that apoB is more predictive of CAC progression than LDL-C. In addition, we found that the discordant high-risk group (high apoB/low LDL-C) had the highest prevalence of hypertension and diabetes, whereas the occurrence of these comorbidities was lowest in the discordant low-risk group (low apoB/high LDL-C). 


\begin{tabular}{l} 
Table 4. ApoB and LDL-C Levels and Their Concordance/Discordance in Relation to Risk of CAC Progression \\
\cline { 2 - 5 } \\
\cline { 2 - 5 }
\end{tabular}

Multivariable model 1 was adjusted for age, year of screening examination, and study center; model 2 was further adjusted for family history of coronary artery disease, education, diabetes, hypertension, smoking status, alcohol consumption, physical activity, and medication for dyslipidemia; model 3 was further adjusted for systolic BP, diastolic BP, BMI, and HDL-C. Abbreviations as in Table 1.

Because a considerable proportion of individuals who experience cardiovascular events have a normal or moderate level of LDL-C, the addition of predictive information about biological markers such as apoB and non-HDL-C is of great interest; ${ }^{16}$ however, there has been controversy about whether apoB would be a more accurate index of coronary event risk than non-HDL-C and LDL-C. In the Emerging Risk Factors Collaboration meta-analysis, the risk estimate of apoB and non-HDL-C and of non-HDL-C and LDL-C were statistically indistinguishable. ${ }^{17}$ One recent meta-analysis showed that apoB is a better predictor of vascular events than non-HDL-C and LDL-C, and one study estimated that targeting apoB rather than LDL-C as a preventive treatment strategy could reduce the number of incident cases among adult residents in the USA by an additional 800,000 over 10 years. ${ }^{18}$ In the Health Professional Follow-up Study using a nested case-control design, patients with CAD had a higher apoB level than the healthy controls, and apoB displayed the strongest association with CAD. ${ }^{19}$ In one prospective cohort study conducted in the USA, women whose level of LDL-C was discordant with apoB $(<$ median LDL-C and $\geq$ median apoB), compared with being concordant $(<$ median LDL-C and <median apoB), were at higher risk of CAD incidence. ${ }^{20}$ Our findings are in line with the results of those studies and extended previous findings, showing that the risk of CAC progression was significantly higher in both the discordant high-risk group (high apoB/low LDL-C) and the concordant high-risk group (high apoB/high LDLC) than in the concordant low-risk group (low apoB/low
LDL-C; reference group). However, there was no significant difference between the discordant low-risk group and the reference group.

Just a few studies evaluated the association of discordance between apoB and LDL-C with subclinical coronary atherosclerosis measured by CAC using a longitudinal study design. In the Coronary Artery Risk Development in Young Adults (CARDIA) study, the plasma level of apoB in young adulthood has been associated with CAC in middle age. ${ }^{4}$ In this study, discordantly high apoB (greater than or equal to the median of apoB and lower than the median of LDL-C) was significantly associated with higher risk for presence of $\mathrm{CAC}$ in midlife, but apoB below the median was not associated with the risk. However, the CARDIA study was limited by the absence of baseline coronary calcium measurements. In the Multi-Ethnic Study of Atherosclerosis (MESA) of 4,623 adults (aged 45-84 years), discordantly high apoB relative to LDL or non HDL-C was modestly associated with CAC progression, which is in line with the present study findings. ${ }^{21}$ Compared to the MESA group, our study population was younger and the association was stronger, suggesting that a risk of CAC progression related to discordantly high apoB/low LDL-C may be greater in younger compared to older individuals. Recent epidemiological studies supported this notion by showing that the risk of cardiovascular events associated with apoB was greater in younger individuals. 22

Although participants with concordant values of apoB and LDL-C (or non-HDL-C) have a likelihood of CAC progression proportional to their concentrations, the risk 
of CAC progression was significantly higher in those with high apoB/low LDL-C, but not in those with low apoB/ high LDL-C. The difference in the risk of CAC progression among discordant participants was consistent with the previous literature and explained by the notion that LDL particles were smaller and denser in the high apoB group than in the low apoB group, leading to higher risk of initiation and maturation of atherosclerotic plaque. ${ }^{23}$ These observations can also be accounted for by elevated remnant cholesterol, which is the cholesterol content of TGrich lipoproteins (IDL and VLDL in the fasting state) and a causal risk factor for a coronary event. ${ }^{24}$ Our study provides a pathological basis for linking discordantly high apoB/low LDL-C with an increased risk of CAC progression. In addition, it is increasingly recognized that obesity and insulin resistance contributing to this process can also lead to production of cholesterol-depleted LDL particles through modulation of cholesterol ester transfer protein driven by an increase in VLDL relative to LDL..$^{25,26}$ Our study indeed showed that participants with discordantly high apoB (cholesterol-depleted LDL particles) had the highest levels of obesity and insulin resistance, suggesting that these phenotypes are related to modulation of cholesterol ester transfer protein.

Several limitations should be considered when interpreting our findings. First, we used CAC scores instead of CAD events as the outcome; however, the association between CAC and CAD events has been shown in previous studies. ${ }^{27}$ Second, study participants were notified of the initial CAC results, which may have contributed to modifying behavior in a healthy direction. As a result, the effect of discordance of apoB and LDL-C on CAD may be underestimated. Third, the follow-up period is relatively short compared with the previous study (25 years). ${ }^{4}$ Significant progression of coronary artery calcification has been shown to occur within approximately 3-6 years. ${ }^{27,28}$ Fourth, our study findings might be underestimated due to a potential healthy worker effect. This effect is a phenomenon in which employees have lower morbidity and mortality compared to the general population because healthy workers tend to remain employed. ${ }^{29}$ Although our study sample comprised mostly employees, this effect is likely to emerge in older populations with more common and symptomatic conditions compared with younger populations. ${ }^{30}$ Our study participants were young and middle aged and free of diagnosed cardiovascular disease. Therefore, the healthy worker effect was less likely to affect our results. Finally, our results were based on a sample of relatively healthy young Korean adults comprised of men and may not be generalizable to other populations.

The major strengths of our study were the large sample size, the use of high-quality data collection methods to obtain a detailed panel of potential confounders and mediators, and the use of a relatively healthy population without a history of CVD. Furthermore, the advantage of this study compared to previous studies is the presence of a baseline CAC score, which allows for calculation of estimated change in coronary calcium score. Thanks to those factors, the association of lipid profiles (including apoB and LDL-C) with the presence and progression of CAC was less likely to be confounded by comorbidities or medication use.

\section{Conclusions}

In conclusion, we found that discordantly or concordantly high apoB was associated with increased risk of CAC presence and its progression, independent of LDL-C. These findings suggest that screening of apoB in healthy middleaged persons may help predict progression of coronary atherosclerosis.

\section{Authors' Contributions}

C.-W.K., Y.C., S.R., and H.S. planned and designed the study and directed its implementation. C.-W.K. made substantial contribution to the study conception and design. C.-W.K., Y.C., and S.R. were responsible for data acquisition and analysis. C.-W.K. and S.H. wrote the draft of the article, and all authors approved the final article for publication.

\section{Disclosures}

Potential conflict of interest: Nothing to report. The name of the ethics committee: The Institutional Review Board of Kangbuk Samsung Hospital.

\section{References}

1. Olofsson SO, Boren J. Apolipoprotein B: A clinically important apolipoprotein which assembles atherogenic lipoproteins and promotes the development of atherosclerosis. J Intern Med 2005; 258: $395-410$.

2. Barter PJ, Ballantyne CM, Carmena R, Castro Cabezas M, Chapman MJ, Couture P, et al. Apo B versus cholesterol in estimating cardiovascular risk and in guiding therapy: Report of the thirty-person/ten-country panel. J Intern Med 2006; 259: 247-258.

3. Sniderman AD, St-Pierre AC, Cantin B, Dagenais GR, Despres JP, Lamarche B. Concordance/discordance between plasma apolipoprotein B levels and the cholesterol indexes of atherosclerotic risk. Am J Cardiol 2003; 91: 1173-1177.

4. Wilkins JT, Li RC, Sniderman A, Chan C, Lloyd-Jones DM. Discordance between apolipoprotein B and LDL-cholesterol in young adults predicts coronary artery calcification: The CARDIA Study. J Am Coll Cardiol 2016; 67: 193-201.

5. Sniderman AD, Lamarche B, Contois JH, de Graaf J. Discordance analysis and the Gordian Knot of LDL and non-HDL cholesterol versus apoB. Curr Opin Lipidol 2014; 25: $461-467$.

6. Boekholdt SM, Arsenault BJ, Mora S, Pedersen TR, LaRosa JC, Nestel PJ, et al. Association of LDL cholesterol, non-HDL cholesterol, and apolipoprotein B levels with risk of cardiovascular events among patients treated with statins: A meta-analysis. JAMA 2012; 307: 1302-1309.

7. Mora S, Glynn RJ, Boekholdt SM, Nordestgaard BG, Kastelein JJ, Ridker PM. On-treatment non-high-density lipoprotein cholesterol, apolipoprotein B, triglycerides, and lipid ratios in relation to residual vascular risk after treatment with potent statin therapy: JUPITER (justification for the use of statins in prevention: an intervention trial evaluating rosuvastatin). $\mathrm{J}$ Am Coll Cardiol 2012; 59: 1521-1528.

8. Ference BA, Kastelein JJP, Ginsberg HN, Chapman MJ, Nicholls SJ, Ray KK, et al. Association of genetic variants related to CETP inhibitors and statins with lipoprotein levels and cardiovascular risk. JAMA 2017; 318: 947-956.

9. Pencina MJ, D’Agostino RB, Zdrojewski T, Williams K, Thanassoulis G, Furberg CD, et al. Apolipoprotein B improves risk assessment of future coronary heart disease in the Framingham Heart Study beyond LDL-C and non-HDL-C. Eur J Prev Cardiol 2015; 22: $1321-1327$.

10. Greenland P, Blaha MJ, Budoff MJ, Erbel R, Watson KE. Coronary calcium score and cardiovascular risk. J Am Coll Cardiol 2018; 72: 434-447.

11. Budoff MJ, Young R, Lopez VA, Kronmal RA, Nasir K, Blumenthal RS, et al. Progression of coronary calcium and incident coronary heart disease events: MESA (Multi-Ethnic Study of Atherosclerosis). J Am Coll Cardiol 2013; 61: 1231-1239.

12. Kim CW, Chang Y, Zhao D, Cainzos-Achirica M, Ryu S, Jung HS, et al. Sleep duration, sleep quality, and markers of subclinical arterial disease in healthy men and women. Arterioscler Thromb Vasc Biol 2015; 35: 2238-2245. 
13. Chang Y, Yun KE, Jung HS, Kim CW, Kwon MJ, Sung E, et al. $\mathrm{A} 1 \mathrm{C}$ and coronary artery calcification in nondiabetic men and women. Arterioscler Thromb Vasc Biol 2013; 33: 2026-2031.

14. Agatston AS, Janowitz WR, Hildner FJ, Zusmer NR, Viamonte M Jr, Detrano R. Quantification of coronary artery calcium using ultrafast computed tomography. J Am Coll Cardiol 1990; 15: $827-832$.

15. Berry JD, Liu K, Folsom AR, Lewis CE, Carr JJ, Polak JF, et al. Prevalence and progression of subclinical atherosclerosis in younger adults with low short-term but high lifetime estimated risk for cardiovascular disease: The coronary artery risk development in young adults study and multi-ethnic study of atherosclerosis. Circulation 2009; 119: $382-389$.

16. Genest J Jr, McNamara JR, Ordovas JM, Jenner JL, Silberman SR, Anderson KM, et al. Lipoprotein cholesterol, apolipoprotein A-I and B and lipoprotein (a) abnormalities in men with premature coronary artery disease. J Am Coll Cardiol 1992; 19: 792 802 .

17. Emerging Risk Factors Collaboration; Di Angelantonio E, Sarwar N, Perry P, Kaptoge S, Ray KK. Thompson A, et al. Major lipids, apolipoproteins, and risk of vascular disease. JAMA 2009; 302: $1993-2000$

18. Sniderman AD, Williams K, Contois JH, Monroe HM, McQueen MJ, de Graaf J, et al. A meta-analysis of low-density lipoprotein cholesterol, non-high-density lipoprotein cholesterol, and apolipoprotein B as markers of cardiovascular risk. Circ Cardiovasc Qual Outcomes 2011; 4: 337-345.

19. Pischon T, Girman CJ, Sacks FM, Rifai N, Stampfer MJ, Rimm EB. Non-high-density lipoprotein cholesterol and apolipoprotein $\mathrm{B}$ in the prediction of coronary heart disease in men. Circulation 2005; 112: 3375-3383.

20. Mora S, Buring JE, Ridker PM. Discordance of low-density lipoprotein (LDL) cholesterol with alternative LDL-related measures and future coronary events. Circulation 2014; 129: 553-561.

21. Cao J, Nomura SO, Steffen BT, Guan W, Remaley AT, Karger $\mathrm{AB}$, et al. Apolipoprotein B discordance with low-density lipoprotein cholesterol and non-high-density lipoprotein cholesterol in relation to coronary artery calcification in the Multi-Ethnic Study of Atherosclerosis (MESA). J Clin Lipidol 2020; 14: 109121.e5.
22. Sniderman $A D$, Islam $S$, McQueen $M$, Pencina $M$, Furberg CD, Thanassoulis G, et al. Age and cardiovascular risk attributable to apolipoprotein B, low-density lipoprotein cholesterol or nonhigh-density lipoprotein cholesterol. J Am Heart Assoc 2016; 5: $\mathrm{e} 003665$.

23. Langlois MR, Chapman MJ, Cobbaert C, Mora S, Remaley AT, Ros E, et al. Quantifying atherogenic lipoproteins: Current and future challenges in the era of personalized medicine and very low concentrations of LDL cholesterol. A consensus Statement from EAS and EFLM. Clin Chem 2018; 64: 1006-1033.

24. Varbo A, Nordestgaard BG. Remnant lipoproteins. Curr Opin Lipidol 2017; 28: 300-307.

25. Cromwell WC, Otvos JD, Keyes MJ, Pencina MJ, Sullivan L, Vasan RS, et al. LDL particle number and risk of future cardiovascular disease in the Framingham Offspring Study: Implications for LDL management. J Clin Lipidol 2007; 1: 583-592.

26. Varvel SA, Dayspring TD, Edmonds Y, Thiselton DL, Ghaedi L, Voros S, et al. Discordance between apolipoprotein B and low-density lipoprotein particle number is associated with insulin resistance in clinical practice. J Clin Lipidol 2015; 9: 247-255.

27. Polonsky TS, McClelland RL, Jorgensen NW, Bild DE, Burke GL, Guerci AD, et al. Coronary artery calcium score and risk classification for coronary heart disease prediction. JAMA 2010; 303: $1610-1616$.

28. Kim S, Chang Y, Cho J, Hong YS, Zhao D, Kang J, et al. Life's simple 7 cardiovascular health metrics and progression of coronary artery calcium in a low-risk population. Arterioscler Thromb Vasc Biol 2019; 39: 826-833.

29. Li CY, Sung FC. A review of the healthy worker effect in occupational epidemiology. Occup Med (Lond) 1999; 49: 225-229.

30. Thygesen LC, Hvidtfeldt UA, Mikkelsen S, Bronnum-Hansen H. Quantification of the healthy worker effect: A nationwide cohort study among electricians in Denmark. BMC Public Health 2011; 11: 571 .

\section{Supplementary Files}

Please find supplementary file(s); http://dx.doi.org/10.1253/circj.CJ-20-0692 